\title{
Across the Divide: Two Organisations Form a Virtual Team and Codevelop a Product
}

\author{
L. Levine ${ }^{1}$, G. Syzdek ${ }^{2}$ \\ ${ }^{\prime}$ Software Engineering Institute, Carnegie Mellon University, Pittsburgh, PA. Tel. (412) 268- \\ 3893, Fax (412) 268-5758, ll@sei.cmu.edu \\ ${ }^{2}$ Red Sky Interactive, Houston, TX. Tel (713) 354-1541, Fax (713) 354-1600, \\ gsyzdek@redsky.com (formerly with Computer Associates Inc.)
}

Key words: collaboration, collaborative technology, virtual teams

\begin{abstract}
This paper takes a socio-technical perspective and presents preliminary findings from a study of a partnership between two organisations-where a virtual team, made up of members from both organisations, came together to codevelop a product. The authors assess what is gained and what is lost in substituting technology for the traditional (same place, same time) working environment and share lessons learned about the use of collaborative technology and processes.
\end{abstract}

\section{INTRODUCTION}

More and more organisations are requiring team members to work together in cyberspace-whether with employees who are geographically dispersed or in partnership with other organisations-in order to accomplish a mutual goal. These collaborative working relationships are about people working together, about sharing information, and about leveraging assets. Organisations are setting collaborative technology in place to help teams work together while reducing travel, speeding review cycles, and lowering costs in order to leverage knowledge.

From a research perspective, teamwork and collaboration technologies have been separately investigated. Relatively few studies have been 
conducted with emphasis on the teamworking aspects of a distributed working environment. We know a great deal about teams: about how members interact and team characteristics, including information on small group communication, position, roles, subgroups, influence, etc. (Henry \& Hartzler, 1998; Lipnack \& Stamps,1997; Sibbet \& Drexler,1994; Wellins, Schaaf \& Harper Shomo, 1994; Isgar, 1993). We also know quite a lot about the technical aspects of collaboration technology (i.e., tools and their functionality) that enable distributed working environments (Kock, 1999; Ackerman, 1996; Grudin et al, 1996; Bannon \& Schmidt, 1989). Too little research has tackled the interplay between these topics, namely the social dimensions of distributed work and collaboration technology in use-what we call the socio-technical system. ${ }^{1}$ Too often, studies fail to address the practical implications of collaborative activity. Meantime, many organisations are at a loss for proven practice in this new area. We intend to address both of these gaps - to take a socio-technical perspective on distributed work and to offer practical information for others, based on our collaboration experience.

In this paper, we present preliminary findings from a study of a partnership between two organisations - where a virtual team, made up of members from both organisations, came together to codevelop a product. This work product developed by the team is a defined process for technology analysis, adoption, and installation, and is briefly discussed in the next section. The discussion that follows is based upon our experience using collaboration technology during a two-year project. By taking a sociotechnical perspective, we emphasise how team members from distributed working environments joined together to solve problems-including to what extent freely and easily available collaboration technology could efficiently support the work. Based on our experience, we assess what is gained and what is lost in substituting technology for the traditional (same place, same time) working environment. We share lessons learned and provide some of our proven practices for using collaboration technology. A more comprehensive report may be published at a later date. The current analysis is considered preliminary for several reasons:

- data analysis is not complete: (a) "collaboration forms" developed and used by project team members to capture data, through time, are not included (b) observers' findings are not presented in detail

- review of the literature is incomplete

- full search for explanatory frameworks (on collaborative activity) for mapping against data is incomplete

- research method, whether experience report, action research (Baskerville $\&$ Wood-Harper, 1996), or qualitative reflexive/narrative study is not treated in depth. 
The sections that follow include the purpose and rationale for the study, project description (high level), discussion of models and frameworks for collaboration activity, preliminary findings, and conclusions.

\section{PURPOSE AND RATIONALE}

By collecting data on the collaboration experience, as we were engaged in the process itself, we gained insight into barriers and enablers associated with the team's real world use of collaborative processes and technologies. The approach allowed us to concentrate on developing the product while not losing sight of the lessons associated with collaborative work. We had two objectives in mind:

1. to codify our findings about benefits and problems in using collaboration technology

2. to then distil "collaboration technique" information (procedural knowledge) from those findings, and to encapsulate that technique in the product we were building.

We achieved the first objective- - to capture and document our learning about collaboration processes and technology - but the development schedule did not allow for the creation of a collaboration technique as part of the final product. However, much of what we learned about collaboration and teaming did find its way, directly and indirectly, into the product we were building. In direct fashion, these discoveries influenced our perspective and deliberate solution development. More indirectly, all products have cultural assumptions built in, and INTRo was no exception as an artefact "inscribed" with the social and political circumstances of its creation.

A brief explanation about the nature of our partnership is in order. The primary goal of our organisations' working relationship was to leverage our respective assets to produce the IDEAL-Based New Technology Rollout process, INTRo: a defined process for technology analysis, adoption, and installation. The partnership between the organisations was established when two of its members recognised the benefit that could be achieved in working together. Both organisations had performed work in the software process improvement arena. The Software Engineering Institute (SEI) is a research and development centre, sponsored by the Department of Defense, with a mission to improve the state of the practice of software engineering. The SEI has developed an improvement model that offers an effective high-level approach to adopting processes, methods, and tools. Platinum technology inc, ${ }^{2}$ (Platinum) was a software vendor that, among 165 commercially sold products, developed a tool for project and process management, including a best practices process library. Platinum also had a process model that its 
implementation managers used to deploy the project/process management tool at customer sites. (Platinum technology, Inc. was acquired by Computer Associates, Inc. in 1999. Henceforth, Computer Associates is referred to as CA.) Together, the organisations sought to integrate the strengths of both models and to test their applicability in new technical areas. The work drew upon SEI know-how on software process improvement and change management and Platinum's technical expertise in architecting processes and practical experience rolling out new technologies in customer and partner organisations.

Both organisations also supported the secondary goal to codify the lessons learned about the use of collaborative technology. From the start, it was clear that INTRo had to be developed in a distributed environment, by team members in Pittsburgh and Houston, and with heavy reliance on collaboiation technologies. Using more advanced forms of collaborative technologies was a relatively new experience for both organisations. The SEI had done research on supporting collaborative processes, but the focus had been on process formalisation and automation (Heineman et al, 1994; Christie et al, 1996). Platinum's teams collaborated internally and with business partners. However, they usually did so asynchronously using the standard set of communication mechanisms of email, fax and telephone. Email was also used to support threaded discussion, review, and feedback.

We recognised that the joint effort would stretch the limits of ordinary collaboration. The literature on collaboration discusses information sharing and communication. However, the rich codevelopment we were envisioning, involving synchronous and asynchronous work, aimed at producing a high quality, fully integrated product would push the boundaries of conventional collaboration. ${ }^{3}$

Our work together would include planning, design, development, decision making, problem solving, project management, technical reviews, negotiations, and documentation production. For our newly created team, the use of collaborative technology to codevelop a product in real time was a pioneering endeavour for our organisations. Our codevelopment, done through distant boundaries, was one of shared creation: a creation resulting from group understanding that no one previously held or could have created through an individual effort.

\subsection{Approach}

We have noted that there is a lot of material available from the fields of Communication and Organisational Development on teaming and group dynamics. Increasingly, attention is also being paid to the culture of organisations (Schein, 1999; Schein, 1996; Constantine, 1993). In Europe, 
especially in Scandinavia, more significant work has been done on sociotechnical issues in computer supported cooperative work (CSCW). Too often, the research community has pushed out a host of studies, largely academic and more limited in nature, often incorporating "toy tasks" (Kraut, Miller \& Siegel, 1996; Redmiles, 1993; Carstensen, 1997; Stebbins, 1993; Albrechtsen \& Jacob, 1998). The challenge is two part: (1) to bring the socio-technical perspective to research and practice, using multiple methods of inquiry to understand technology in use (2) to conduct and disseminate studies that are real world, where organisations and institutions can straightforwardly understand the implications and lessons and clearly act upon the same. We characterise this as a whole systems approach in the spirit of soft systems and living, complex adaptive systems (Checkland, 1999a; Checkland, 1999b; Gharajedaghi, 1999; McMaster, 1996; Wheatley, 1999).

Why do so few research studies, in the areas above and in information technology, fail to see the importance of such integration? It is clear that the work people do is influenced by the systems they use or choose not to use; it's also clear that systems design, for example participatory design, is influenced and benefits from information from system users. Technology maturation, including development and deployment, involves the mutual adaptation of technology and organisation (Leonard Barton, 1988a; Leonard Barton, 1988b). Nonetheless, integrative approaches are not common (McMaster, Vidgen \& Wastell, 1998) nor do we often see an applied research effort that traces a real world project, developing a product.

The present inquiry strives to understand the dynamics at work in a complex collaboration and to offer practical information about some of our proven practices. Our project was not about pure experimental use, about just playing with "cool tools." The effort, from the start, had a strategic pragmatism about it - the stakes were high, the goals and expectations were aggressive, and the use of new technology was consistently in service of the work. We hope this paper contributes alongside much of today's more theoretical work by providing practical examples of collaboration technology in use.

\section{PROJECT DESCRIPTION}

This paper operates at several logical levels concurrently, to consider (1) a socio-technical perspective on collaborative practice (2) our particular organisational and technical drivers and constraints (3) the task at hand, namely the codevelopment of INTRo (4) our findings with respect to the above levels. 
We ask the reader's patience with the presentation order for the next three sections-on project description, models and frameworks for collaboration, and on preliminary findings. Each of these sections might be presented first and used to frame the subsequent discussion. In addition, the subjects necessarily overlap. In essence, we are trying to succinctly describe what we have found on this project in terms of our collaborative workwhich is a topic unto itself and one that touches all three sections.

In the project description and collaboration frameworks sections, we provide just enough contextual information to make the preliminary findings meaningful to the reader. The project description includes the timeline for the project and information on the composition of the virtual team. We also briefly comment on how we captured data on the collaboration process as it was occurring.

\subsection{Project Start-Up}

A process design workshop, January 1998, was held to kick-start the development process. The workshop allowed the opportunity for five core team members to meet each other and to discuss:

- principles upon which the INTRo process would be based

- scope of the process, roles and responsibilities

- major products

- overall structure of the process

- business case, risks

- schedule, process development process for INTRo

The workshop included an overview of the process development approach and tools (used at Platinum) which would provide the authoring environment. The workshop also allowed time to discuss the collaborative working arrangements: how the work would be done, collaboration processes and technology, infrastructure, and data capture on collaboration. We knew that in order for the team to successfully develop INTRo, we had to rely heavily on collaborative technologies. Team needs were noted, including the following:

- Develop the process in real time

- Edit data in shared applications in real time

- Share the flow of information among team members

- Build trust and camaraderie among virtual team members

- Have occasional face-to-face meetings with team members 


\subsection{Team Composition}

Initially, in 1998, the core team consisted of two technical staff from SEI and two from Platinum. The extended team included contributors, reviewers, pilot participants, making up a total of five members. In 1999, the curs team was reduced to one technical staff from SEI and one from Platinum. Extended team members continued to play a critical role in review and a greater role in pilot activity. A total of three members participated in 19992000 .

\subsection{Timeline}

A high-level timeline of INTRo development, recording key activities appears in Figure 1. This timeline reflects major milestones in actual development, not from plans. In addition, twelve face-to-face meetings took place between Sept 1997 and October 1999. These meetings, which had a strong reinforcing effect, included technical interchange, alpha reviews, process overviews at the pilot site, walkthrough reviews, conference presentations, and requirements sessions (focused on change requests and enhancements).

It is worth noting that initial estimates for INTRo, related to scope, size, and schedule, projected a one-year effort. During the course of development, it became evident that INTRo was a larger and more complex product than originally envisioned. In addition, since we were developing process guidance which users would be implementing, we felt it would be important to pilot INTRo. We adjusted our plans. At the end of year one, (1998), we would produce a beta version, for trial or pilot use. The following year, we would deliver the final version 1.0, which would incorporate as many changes as possible resulting from pilot feedback, not simply comments from walkthroughs and reviews. 


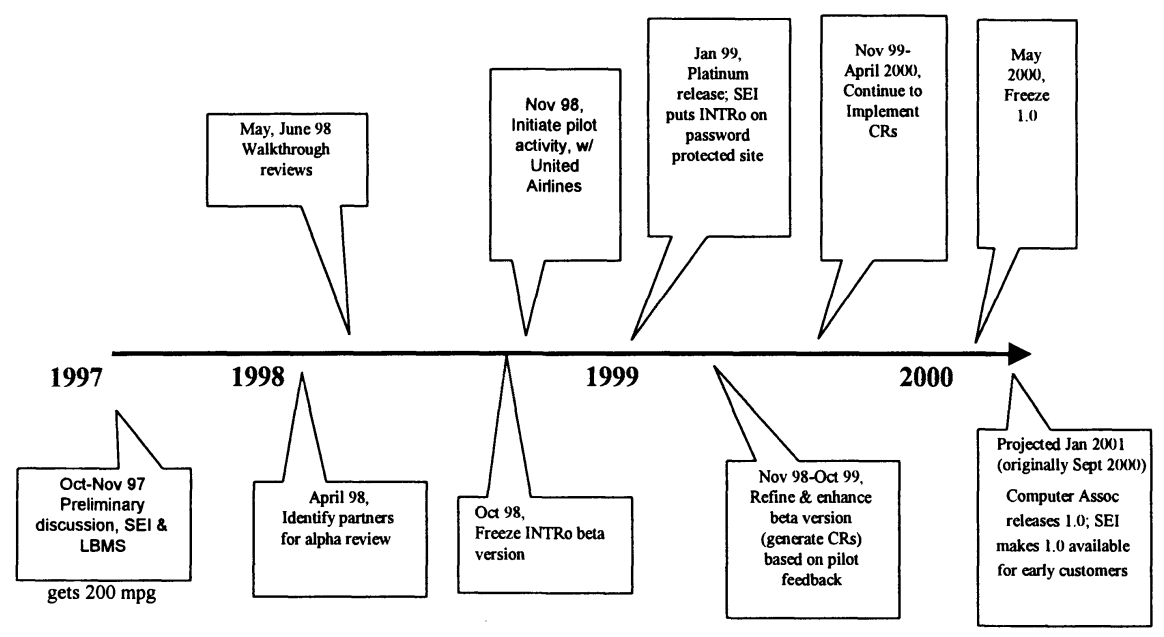

Figure 1. Major Milestones in Development

\subsection{Data Capture}

During project start-up, discussion of collaboration was key: how would the team work together to codevelop INTRo and to codify lessons on collaboration and virtual teams? Team members agreed to

- capture data through the use of a "collaboration form," recognising that these forms would evolve according to need

- invite observers to virtual meetings, for several sessions. This would allow multiple perspectives on the collaboration activities. Observers would be asked to record and share their observations. (One observer was an expert in Instructional Design; a second observer specialised in group dynamics, teaming, and change management.)

- document and publish findings in white papers, technical reports, and articles

- based on experience and lessons, attempt to build a "collaboration technique" to be encapsulated in INTRo

Preliminary decisions were made about the different technologies needed to support asynchronous and synchronous work. As a result, examples of the asynchronous technologies that project members selected and used included email with/without attachments, voice mail, and fax. Typical synchronous technology chosen and used included telephone, videoconference, and a desktop conference tool. Real-time meetings involved multi-party interactive sessions (easily available at both organisations' desktop workstations) where team members communicated 
with each other directly, using Microsoft's NetMeeting. This conferencing tool allowed participation in cooperative interactive visualisation and display of data. The team was able to get significant mileage out of NetMeeting. This single application enabled sharing and collaboration with a host of applications, in particular, the process authoring tool being used to define INTRo, file transfer, and whiteboard. Further elaboration is found in the sections on collaboration models and preliminary findings.

\section{MODELS AND FRAMEWORKS FOR COLLABORATION ACTIVITY}

The framework below identifies different types of collaboration, in terms and categories and distinctions commonly used in the computer supported collaborative work (CSCW) literature. ${ }^{4}$ Technologies that support collaboration are indicated-according to whether the work is performed by individuals at the same time (synchronously) or at different times (asynchronously). In addition, distinctions are drawn between activities that facilitate communication and information sharing. Along with this labelling comes some inaccuracy; it is easy to see places of overlap. For example, communication is an essential ingredient in information sharing, even though we may want to set information sharing apart since it appears more interactive in nature, suggesting dialogue or back and forth exchange. Types of collaboration and mechanisms used by the team are indicated with a check mark $(\sqrt{ })$ in Table 1.

We believe that communication and information sharing fall short as labels to account for the nature of our collaboration experience, which involved significant joint activity. Many of the technologies used for information sharing were also used to support real-time codevelopment. This raises the question of whether existing frameworks are sufficient to delineate the full range of activities that people engage in when they work together. To better capture our experience, we would add "codevelopment" as a category. Even then, we would require additional models or descriptions to characterise the richness of our cooperative work-qualitative issues, such as effectiveness and performance; and in terms of what worked well, how we stitched things together, and why we did what we did. Such concerns are critical for deeper understanding of collaborative practice involving the use of technology.

A collaborative working environment is about people working together, about sharing information, and making decisions based on knowledge transfer and dialogue. In the description of our collaborative arrangement, we show how team members from distributed working environments joined 
together to solve problems and to what extent the collaboration could be supported through computer technology.

Table 1. Types of Collaborations and Mechanisms Adapted from Grudin et al, 1996.

\begin{tabular}{|c|c|c|}
\hline $\begin{array}{l}\text { COLLABORATION } \\
\text { TYPE }\end{array}$ & COMMUNICATION & $\begin{array}{l}\text { INFORMATION } \\
\text { SHARING }\end{array}$ \\
\hline ASYNCHRONOUS & $\begin{array}{l}\text { Email + attachments } \sqrt{ } \\
\text { Voicemail } \sqrt{ } \\
\text { Videomail }\end{array}$ & $\begin{array}{l}\text { Discussion groups } \\
\text { Group Editing } \\
\text { (shared software revision } \\
\text { control) }\end{array}$ \\
\hline $\begin{array}{l}\text { SYNCHRONOUS (REAL- } \\
\text { TIME) }\end{array}$ & $\begin{array}{l}\text { Chat, } \\
\text { Telephone, } \sqrt{ } \\
\text { Video conference } \sqrt{ }\end{array}$ & $\begin{array}{l}\text { Application Sharing } \\
\text { And } \\
\text { Applications that Share } \\
\text { Co-development } \\
\text { (NetMeeting: Share } \\
\text { Applications \& Collaborate, } \\
\text { File Transfer, and occasional } \\
\text { use of whiteboard; } \\
\text { applications shared include } \\
\text { Word, PE, PowerPoint) }\end{array}$ \\
\hline
\end{tabular}

Table 2 further illustrates the time and place distinctions for synchronous and asynchronous work.

Table 2. Time/Place Distinctions and Example Mechanisms

\begin{tabular}{lll}
\hline Time & Place & Example \\
\hline Same & Same & Face to Face Meetings \\
Different & Different & Asynchronous: email, fax, \\
& & voice mail \\
Same & Different & Virtual Meeting \\
& & Environments, Synchronous, \\
& & Telephone, video \\
& & conferencing, chat \\
Different & Same & Serial Working \\
\hline
\end{tabular}

Sometimes, the terms loosely coupled and tightly coupled are also used to distinguish between synchronous or asynchronous work.

Loosely Coupled

Asynchronous

Email, voice mail
Tightly Coupled

Synchronous (real-time)

lephone, audio/video conferencing, chat

Collaborative applications, involving loose coupling, are characterised by work on shared objects at different times. Conversely, team members that 
use tightly coupled collaboration work at the same time as others on a common shared workspace (Mitchell \& Graham, 1995). Responsiveness and notification are critical in tightly coupled collaborative systems. "Responsiveness refers to the immediacy of local system reaction to local user actions, whereas notification refers to the immediacy of remote propagation of local user actions... When dealing with tightly coupled systems, users should be notified of other users' actions with as little delay as possible" (Mitchell \& Graham, 1995). Tightly coupled applications are essential with real-time codevelopment activity. When NetMeeting, a tightly coupled collaborative application, begins to break down by delaying responsiveness and notification, this hinders the execution of tasks, whether brainstorming, simple free flow of ideas, design, drafting, or composition.

\section{PRELIMINARY FINDINGS}

For purposes of structure, we have categorised the preliminary findings according to whether they relate to people, process, or technology issues.

\subsection{People}

This category includes information on the attributes of individuals and teams, as well as on organisational culture and professional subcultures. Some researchers treat people and organisational concerns separately-a distinction that may prove important in a subsequent full report. For the present, we use the single category of People. 


\subsubsection{Team Characteristics}

The characteristics of the core team are noteworthy, especially the high levels of commitment, energy, focus, and communication. These characteristics were there almost from the beginning, which may account for how like-minded individuals identified a need and worked as self-starters, or to coin a phrase, "team-starters" to lobby their respective organisations, band together and create a joint effort. Some would describe this behaviour as that of a high performing team (Sibbet \& Drexler, 1994). A reinforcing effect also operated. High energy fed team interaction and resulted in a "can do" attitude and strong productivity; in turn, the positive attitude and productivity generated increased energy and commitment.

Since this codevelopment experience is being told from the team's perspective, we recognise that readers may or may not choose to accept our characterisation of the team at face value. In self-reporting, directness and reliability are trade-off concerns. 
One observer offered the following description of the team:

- like minded

- found middle ground

- jelled together

- small group

- same vision

- personalities of team members plays a role

- high performance team qualities

- self managing

Over time, there were changes in the composition of the team. For example, in the Spring of 1999, a core team member from Platinum left the project and the organisation. As a result, another CA member picked up additional responsibility. Initially, this adjustment worked well; however, six months later, the same team member was struggling to meet her various responsibilities and commitments. As a result, she had to cut back time spent on INTRo development.

Two SEI contributor/reviewers were involved in the first year of development (1998), and different reviewers came on board for the second year (1999). These changes were relatively smooth. In February 2000, two months before the final product freeze (targeted for April 2000), the CA lead member left the project and the organisation, handing off to a colleague from CA. This disruption was more significant, resulting in several months project delay. The new team member had deep knowledge of the authoring environment and tooling but limited understanding of the INTRo product. (Original target release for INTRo was September 2000; release is now projected for January 2001.) Overall, the team responded, accommodated and moved forward as best it was able.

It is worth noting that the duration of the codevelopment effort was longer than most collaborative projects. This allowed the team to cycle through the processes of forming, storming, norming, and performing, with the ability to continue to perform through time, leveraging team growth and learning. Conversely, as noted, the project endured two acquisitions-as LBMS was acquired by Platinum technology Inc. in 1998 and then, roughly one year later in 1999, as Platinum was acquired by CA. These acquisitions brought different degrees of instability to project work and were, at minimum distracting and at maximum more disturbing. For approximately 3-4 months in 1999, the uncertainty and cultural and political changes surrounding the second acquisition affected morale and significantly reduced productivity. 


\subsubsection{Mental Models}

Team members constructed a shared a mental model of the project. They had a common vision, values, preferences, and work styles. Some differences emerged-between the views and practices of the industry organisation (Platinum/CA) and the R\&D institute (SEI). However, even while experiencing dissonance, team members placed value and store in their divergent perspectives, realising that the variation would balance and improve the work products and provide a richer team mix. Different perspectives were most evident about time to market and project management. The industry perspective favoured the earliest possible release of INTRo whereas the Institute perspective wanted to ensure that INTRo was accurate, fully tested, and complete. A compromise was struck. Thus, Platinum released the initial version of INTRo in its product suite (January 1999). SEI chose to consider this a beta version, and made it available for limited access and distribution on a password-protected site.

In terms of project management, Platinum's approach was more detailed and rigorous than SEI's. We speculated that the different approaches to project management reflected differences between academia and industry practice, and perhaps also where these two organisations fell along a technology development life cycle. The SEI conducts applied research on the maturation of new technology; Platinum does advanced development, building commercial products and offering consulting services.

In addition to team members explicitly and deliberately valuing difference, the parties shared an overall pragmatism. This pragmatic bent helped to navigate difference and still allowed the team to optimise according to the strengths and expertise of the parties. Through strategic thinking and an emphasis on alignment, reuse and dual use, the team sought to maximise the impact and benefit of its development efforts. Planning and designing for data capture on the team's collaboration experience, during the course of codevelopment, is an apt example.

\subsubsection{Sponsorship}

At SEI, sponsorship of the project was sustained throughout, although the effort had some of the attributes of a small skunk works or outlaw project, directly supported (sponsored and championed) by a senior manager. On the industry side, for several months during the acquisition (of Platinum by CA in 1999) support waned, when the project lost a key team member who was also a champion. As a result, industry-side sponsorship was fragile and undetermined for a time and needed to be rebuilt. Gradually, support was reestablished when the new lab manager came on board at CA. This manager 
proved a strong advocate and supporter. This was evident even through the last set of staff changes at $\mathrm{CA}$.

\subsection{Processes}

\subsubsection{Establishing Trust}

One issue that cuts across people and process issues is the matter of building trust. This is especially important for collaborative distributed work. Moreover, trust involves levels - being able to trust another to be a good and sincere individual is not the same as being able to trust another's project management skills. The trust that is built on a virtual team is based on a growing sense of the others' experience in the work/subject area, credibility, as well as the ability to make sound judgements. Working at a distance, without much history or direct personal contact, one wonders about collaborators in these ways: Is the message logically sound? Does the information flow? Did the experience she was describing seem sensible or correct, like the right thing to be doing in the situation? Can I relate? If the answers to such questions are reassuring, gradually, through time, trust can be built.

On our virtual team, trust was built early on and for the most part remained high. Only during the tensest times, was trust in question. This happened twice-first, when Platinum was acquired by CA and the project lost a member/champion on the Platinum side in Spring 1999, and second, when the CA lead member left the project and the organisation, in February 2000 , two months before project freeze. On these occasions, team members struggled with balancing the needs of the project with their needs as individuals.

Acquisitions are notorious for raising political and cultural instability and our project was no exception. The effort proceeded through two acquisitions-perhaps in itself a badge of success. At acquisition and merger, matters of individual and organisational trust blur. During the first acquisition (of LBMS by Platinum in 1998), there was a relatively short period of uncertainty. Platinum managers arrived on the LBMS scene early and worked quickly to characterise the company's future direction, integration, and transition. During the second acquisition (of Platinum by CA in 1999), there was more uncertainty for a longer period of time. Integration took longer, most likely because Platinum was only one of the many companies that $\mathrm{CA}$ acquired over a short period of time. The entire team was unsure about what to make of $\mathrm{CA}$ and about future levels of interest and support for our collaborative project, although this was clearly 
more pressing for Platinum/CA staff. At this time, individuals were wary about trusting their employers and what lay ahead. We have already seen that sponsorship was gradually re-established when the new lab manager came on board at CA. This went a far distance in rebuilding trust at the organisational level.

\subsubsection{Maintaining Contact}

Researchers maintain that working relationships require some amount of direct personal contact. We felt this to be true and sought face to face meetings at intervals for this reinforcing effect. As noted, twelve face-to-face meetings took place between Sept 1997 and December 1999, with both parties travelling roughly the same amount of the time. For a few meetings, for example a visit to the pilot site or to a conference, not all team members were present. However, face to face meetings were designed to combine a working session with another engagement, whether it was a technical interchange, review (alpha, walkthrough), requirements session, visit to pilot site, or conference presentation. Only the initial planning visit in November 1997 and the Process Design Workshop, which launched the project, served a single purpose.

\subsubsection{Decision Making}

Decision making processes should not be seen in isolation from the characteristics previously identified: the team's high-performing nature, its common purpose and shared mental models, strong commitment and high levels of trust. No single decision-maker or leader dominated. Rather, because the team as a whole valued the different orientations, perspectives and experiences of its parties, members typically deferred to whichever party had greater expertise with respect to an issue. With project planning and tracking, and process architecture, Platinum/CA provided more steering, whereas the SEI exerted greater influence on research direction, scope, and pilot methods. Open communication, discussion, and negotiation preceded all decision making.

The team never experienced differences of opinion that derived from powerplays, moves for territory or from the management of a group where mistrust leads to fearfulness about grabs for power. In the use of NetMeeting, where power and control issues can surface, as the person sharing the application tends to dominate the virtual meeting, individuals led discussion without any attempts to control the interaction. 


\subsubsection{Project Management}

When managed as a project, codevelopment has higher complexity in a distributed work environment and higher overhead. A fair question we might ask is: how is project management different between conventional and virtual teams? Certainly a key issue concerns trust and the need for new thinking about the role of oversight (Handy, 1995). Virtual teams are much more likely to be successful if they are able to function as autonomous, adaptive, and self-organising entities (Davenport \& Pearlson, 1998).

If we were discussing conventional project management, we would likely consider meeting management under the Process category. However, since our meeting management, with a virtual team, was so tightly connected to the use of technology, we cover this topic in the final section on Technology.

\subsection{Technology}

\subsubsection{NetMeeting as NetWorking}

In many ways, NetMeeting is a misnomer for more intense NetWorking. NetMeetings, in our experience, were far more demanding than conventional meetings. These meetings were extremely intense, without much downtime, with participants closely focused on the computer screen. The kind of tuning out that one might do in a conventional meeting is difficult. Rather, participants listen hard to what is said and for additional cues and tone. This was especially evident in the first 6-9 months of the collaboration and eased, somewhat, after that time.

\subsection{2 "Sensory Distortion"}

One of our observers pointed out that sensory distortion was occurring. (This may be so even when video is in use since the quality is often poor.) In face to face meetings, when the conversation quiets, participants have visual cues to explain what is going on. Someone may be reading or writing on the chalkboard. Facial expressions, tone of voice, and body languages, all of which add depth to the perception in communication, are reduced or lost with NetMeeting, telephone, or email. During our NetMeeting experiences, we were unable to see such cues and we became quite adept at sensing and at managing silence. We offered information about silences, keeping each other posted about what was happening, and we shared our reactions freely and directly. Trust is a critical ingredient in such exchange, especially as the opportunities for misunderstanding may be greater in virtual meetings. 
Somers, Rudman \& Stevens (1997) corroborate our experience. They observe: "[m]eeting protocols emerge with collaborative systems. For example knowing who is present in the meeting, knowing who is controlling what application, knowing what everyone (especially the leader) is doing at any point in time, and making sure that all participants are looking at the same thing. Users rely heavily on the audio channel for this co-ordination and thus spend a significant amount of meeting time performing "coordination overhead" rather than the actual business of the meeting."

\subsubsection{Use of NetMeeting}

The team's use of NetMeeting extended to include:

- Project status meetings

- Demonstrations

- Reviews

- Codevelopment/Production

Project Meetings. These meetings allowed team members to report status related to the development schedule, progress on the work breakdown structure for INTRo, and other project issues. These sessions were extremely focused and demanding. NetMeetings generally lasted about 1.5 hours and were well structured, following agendas that had been created as part of the collaboration forms. These forms were developed to capture the meeting agenda, minutes, and to track problems/barriers identified with the use of specific technologies (most frequently NetMeeting in conjunction with application sharing).

Demonstrations. NetMeeting was used to give numerous demonstrations of the authoring tool environment and/or the current work on the INTRo to upper management and other interested parties of the organisations. Demonstrations were also given to market/communicate about the INTRo process to colleagues who might be interested in piloting INTRo after completion.

Reviews. The project team was attentive to process review checkpoints. Again, NetMeeting played an important role. Informal and formal reviews were held between team members and with upper management and external reviewers. During reviews, INTRo's work breakdown and task descriptions were extensively re-examined, and changes were made online, in real-time. Often, the collaborate function was enabled, allowing edits to be made by remote team members.

Codevelopment/Production. Project deliverables such as project plans and schedules, and the design requirements document were reviewed and edited via NetMeeting and transferred to team members during the session through file transfer capability. Since marketing activities were an essential 
part of the development project, presentation slides and other marketing aides were also reviewed and easily edited online.

Example constraints that the team experienced at times, related to tooling and network performance, include:

- Network performance. There were times when we would lose complete connection during our session. This seemed to occur more frequently while sharing MS Word applications. There were times when the network was very slow at returning data back to the other party's screen (screen refresh). Also, when the collaboration feature was turned on, the screen hesitated while the party was trying to add data. When network performance was extremely slow, frustration levels were high and system tolerance levels were low causing us to abandon the collaborative session. Network performance was generally better during earlier morning NetMeeting sessions (8:00 - 11:00 cst).

- The tool used (NetMeeting) was equipped with audioconferencing capabilities to act as "Internet telephones"; however, the telephone on our desk did a better job. NetMeeting was not sufficient to support high quality audio conference. We used the telephone to bridge the audio connection. (This may be because the modem connection (28.8) is not sufficient.)

- Technical difficulties such as software/network disconnects were disruptive and disturbed concentration. Real-time co-development in our collaborative environment was spent partially in tool learning.

- The model used in our working environment was one in which one user placed a call out to the others or answered a call from other meeting members. We did experience constraints such as secured intranets (firewalls) that did not allow entrance into the public directories, connections.

\subsubsection{Meeting Management}

We have already alluded to the team's need for project and meeting management practices. In part, this derived from the nature of the distributed work to be done by a virtual team and from the team's interest in capturing its lessons on the use of collaboration processes and technology through observation, use of forms, etc. However, as a team, we also became increasingly aware of the critical need for advance planning and organisation in order to work productively for our periods of contact.

Mitchell \& Graham (1995) observe that the "ways in which groups work together on a task can range from highly structured, such as in a board meeting, to very unstructured, such as in a brainstorming session between designers." Our team worked together in a highly structured manner in 
status/review meetings, and in more unstructured fashion for brainstorming sessions between developers. In structured sessions, roles were assigned: leader, observer, scribe, etc. In such situations, the passing of control and access to the shared workspace was planned in advance.

Preliminary work before a NetMeeting involved preparation and structuring the virtual collaborative session. The typical sequence of activities looked like this.

- Team members offer input, by email or discussion, for proposed agenda topics

- A team member (identified) reviews the previous collaboration form and action items, and deferred topics looking for additional agenda topics

- A team member (identified) develops a new collaboration form to include received inputs from other participants and deferred agenda topics from previous collaboration meeting

- Agenda is distributed in advance.

Building agendas in this manner created an increased level of participant involvement and assurance that a member's issues would be a part of the agenda. Occasionally, during a meeting, the team needed additional time on a topic. We adjusted the agenda, deferring other topics to the next meeting date. Typically, the team worked hot issues and those of lesser importance were added as last items on the agenda.

We used the term "scripting" to describe how a discussion leader planned and prepared files to be shared before a NetMeeting began. After we learned how the technology worked, and that screen painting could become slow and cumbersome, we realised that jumping from application to application, and from screen to screen had a negative impact on what is seen, and how quickly, by meeting participants. After a few disjointed and confusing sessions, we settled on a scripting heuristic, encouraging discussion leaders to open files in advance to what it was that they wanted to share and walkthrough. This allowed for more seamless movement through the topics at hand. 


\subsubsection{Video conferencing}

Picture Tel was briefly used during the course of our working relationship. Although tried only once, this tool was used to test/experience the video conferencing technology available at each site. The meeting was called to review presentation slides and finalise a conference preparation that was to be given by one of the team members from each site. Technical difficulties experienced during the video conference meeting included:

- voice transmission delay

- initial use instruction (since this was the first time using the technology, assistance was needed in connecting to each others' site)

It is important to emphasise that the use of the collaboration technology on this project was always in the service of the work. The team was always interested in hearing about different technologies that were available and that might help us downstream, but we were careful about learning curves and making sure we would get a strong return on whatever we were going to pick up. For example, the team discussed the use of Basic Support for Cooperative Work (BSCW), but it was never clear that we needed it or couldn't get the value another way. We were aware that for a larger team, we might have really needed BSCW or some other repository based technology.

\subsubsection{Security}

In interorganisational collaboration efforts, it is important to adopt a more open approach to information sharing having concern for access first and then security where protection is needed at some levels of access. Security firewall prevents interorganisational collaboration. Most collaboration tools are designed to allow access between two parties in common web gateway with an explicit connection through directories. However, in our experience when a firewall security was installed at one site, access to the directories was not possible. The work around for us with using NetMeeting was to connect via tcp/ip address. Security issues surfaced at various points during the life of this project, especially since each organisational acquisition had implications for the technical architecture and infrastructure. More extended discussion of this topic is beyond the scope of this paper. 


\section{CONCLUSION}

Clearly, the work people do is influenced by the systems they use or choose not to use, and systems design is influenced by, and benefits from, information and feedback from system users. Yet, as we have observed too few research studies, especially in the American tradition, in Organisational Development and Communication (on group dynamics, teams) and in Computer Supported Cooperative Work and Information Systems, take an integrative approach to socio-technical issues. We have posed a two-part challenge: (1) to work in the margins of these disciplines using multiple methods of inquiry to understand technology in use (2) to conduct and disseminate studies that are real world, so that organisations and institutions can straightforwardly understand the implications and lessons and purposefully act upon the same.

This was our concern as we set about to explore the dynamics at work in our complex collaboration and to offer practical information on some of our lessons and proven practices. We hope this paper contributes alongside much of today's more theoretical work by providing real practical examples of supporting collaboration technology in use.

Our experience, adapting to the use of collaboration technology and processes, resembles some of the already documented experiences of companies adopting alternative work arrangements for distributed work. "Successful virtual offices require radical new approaches to evaluating, educating, organising, and informing team members." Companies must develop the management approaches that make virtual offices effective; management must rethink "the design of their business processes, and they must examine their control, measurement, and evaluation techniques for these new processes." Distant team members must be well connected with the rest of the business (Davenport \& Pearlson, 1998, pp. 51, 60).

And there are old pitfalls. Too often, especially with tools, we see a premature inclination to jump to a technological solution without paying attention to basics. Development teams are often over eager to automate processes, which are not yet well defined or used in manual operations (Christie et al, 1996). "Doing" computer supported cooperative work or using collaboration technology is not guarantee that contributors are collaborating, in the best sense of the word, or working productively as a team. These tendencies reveal our wishful thinking that adding technological support will magically allow participants to leap frog over a host of requirements. Here, technology can be seen, naively, as a silver bullet, allowing one to side-step consideration of the primary and fundamental ingredients associated with effective work practice. 
Working in a new mode or new environment may turn out to be substantially different than a current baseline operation. But the failure to account for what constitutes good practice as a starting point only guarantees compromised success at the next technological level of complexity. A fool with a tool is still a fool. No technology can compensate for bad practice or substitute for an understanding of fundamentals; however, integrating, experimenting with, and piloting new technologies in practice can help us co-evolve fundamentals and technologies. For these reasons, we underscore the importance of a socio-technical perspective and related knowledge in multiple disciplines and in local practice. Initially, one may focus on collaboration technology and thinking about systems and processes. But in the end, effective learning organizations (Argyris \& Schon, 1996; Brown \& Duguid, 1996; Brown \& Gray, 1995; Lundberg, 1991; Nonaka \& Takeuchi, 1995; Schein, 1997) must come to grips with good practice in teaming, education, sharing information and archiving lessons, and corporate memory-recording and analyzing decision making and related history-for recurring and problematic themes, and all in a manner that is coherent, yet streamlined and accessible.

\section{ACKNOWLEDGEMENT}

The authors thank John Goodenough, Dan Green, and the anonymous reviewers for insightful comments used to improve this paper, and Suzanne Couturiaux for editorial assistance.

\section{NOTES}

1 "A socio-technical system is a system composed of technical and social subsystems. An example for this is a factory or also a hospital where people are organized, e.g. in social systems like teams or departments, to do work for which they use technical systems like computers or x-ray machines." PRINCIPIA CYBERNETICA WEB, Web Dictionary of Cybernetics and Systems, Bernd Hornung's Glossary definitions http://pespmcl.vub.ac.be/ASC/Socio- syste.html

2 The initial discussion about joint work began between the SEI and LBMS Inc. in October 1997. LBMS was acquired by Platinum technology Inc. in 1998, and Platinum technology was acquired by Computer Associates in 1999. General references in this paper are made to Computer Associates, unless a specific asset or activity was associated with Platinum's tools, approach, consulting model, or customer set.

3 Typically, when people talk about collaboration, the processes they have in mind involve some combination of division of labor and/or review and feedback. 
4 Adapted from Grudin, Jonathan, Poltrock, Steven E., and Patterson, John F. (1996). CSCW Overview (tutorial notes). CSCW 96, Computer Supported Cooperative Work Conference, November 16-20, 1996, Boston Mass.

\section{REFERENCES}

Ackerman, Mark. (Ed.) (1996). Proceedings of the ACM 1996 conference on computer supported cooperative work. New York: ACM Press.

Albrechtsen, H. \& Jacob E. (1998). The dynamics of classification schemes as boundary objects in electronic libraries. In Classification in the electronic environment edited by Geoffrey Bowker and Susan Leigh Star. Library Trends, 47(2), Fall 1998, 293-312 (background paper)

Argyris, C. \& Schon, D. A. (1996). Organizational Learning II, Reading, MA.: AddisonWesley

Bannon, L., \& Schmidt, K. (1989). CSCW: Four characters in search of a context, EC-CSCW '89, Proceedings of the First European Conference on Computer Supported Cooperative Work, Gatwick, London, 13-15 September 1989.

Baskerville, R. \& Wood-Harper, T. (1996). A critical perspective on action research as a method for IS research. Journal of Information Technology 11(4), 235-246.

Brown, J. S. \& Duguid, P. (1991). Organizational learning and communities of practice: Toward a unified view of working, learning, and innovation. Organization Science 2(1), 58-82

Brown, J. S. \& Gray, E. S. (1995). After reengineering: The people are the company. Fast Company, Premiere issue, 78-81.

Carstensen, Peter H. (1997). Toward information exploration support for engineering designers. In S. Ganesan (Ed.) Advances in concurrent engineering -CE 97, August 2022, Oakland, Michigan, Technomic, 1997, pp. 26-33.

Christie, A. M., Levine, L., Morris, E. J., Zubrow, D., Belton, T., Proctor, L., Cordelle, D., \& Ferotin, J-E. (1996). Software process automation: Experiences from the trenches. (SEI Technical Report SEI-96-TR-013). Pittsburgh, PA.: Software Engineering Institute.

Checkland, Peter. (1999a) Systems thinking, systems practice: A 30-year retrospective. New York: John Wiley \& Sons.

Checkland, Peter. (1999b). Soft systems methodology: A 30-year retrospective. New York: John Wiley \& Sons.

Constantine, L. L. (1993). Work organization: Paradigms for project management and organization. Communications of the ACM 36(10), 34-43.

Davenport, Thomas H. \& Pearlson, Keri. (1998). Two cheers for the virtual office. Sloan Management Review39 (4), 51-65.

Gharajedaghi, Jamshid. (1999). Systems thinking : Managing chaos and complexity : A platform for designing business architecture. Boston: Butterworth Heinemann.

Grudin, Jonathan, Poltrock, Steven E., \& Patterson, John F. (1996). CSCW Overview

(tutorial notes). CSCW 96, Computer Supported Cooperative Work Conference, November 16-20, 1996, Boston Mass.

Handy, Charles. (1995). Trust and the virtual organization. Harvard Business Review, MayJune, 40-50.

Heineman, G. T., Botsford, J. E., Caldiera, G., Kaiser, G. E., Kellner, M. I., Madhavji, N. H., (1994). Emerging technologies that support a software process life cycle. IBM Systems Journal 33(3), 501-529. 
Henry, Jane \& Hartzler, Meg. (1998). Tools for virtual teams: A team fitness companion. Milwaukee, WI: ASQ Quality Press.

Isgar, Thomas. (1993). The ten minute team: 10 steps to building high performing teams. Boulder, Colorado: Seluera Press.

Kock, Ned. (1999). Process improvement and organizational learning:The role of collaboration technologies. Hershey, USA: Idea Group Publishing.

Kraut, Robert E., Miller, Mark D., \& Siegel, Jane. (196). Collaboration in performance of physical tasks: Effects on outcomes and communication. In Mark S. Ackerman (Ed). Proceedings of the ACM 1996 Conference on Computer Supported Cooperative Work, (pp. 57-66). Boston, Mass: ACM Press.

Leonard-Barton, D. (1988a). Implementation as mutual adaptation of technology and organizations. Research Policy 17,(5) (Oct.), 102-110.

Leonard-Barton, D. (1988b). Implementation characteristics of organizational innovations. Communication Research 15, 603-631.

Lipnack, J. \& Stamps, J. (1997). Virtual teams: Reaching across space, time, and organizations with technology. New York: Wiley.

Lundberg, C. C. (1991). Creating and managing a vanguard organization: Design and human resources lessons from Jossey-Bass. Human Resource Management, 30(1), 89-112.

McMaster, Michael D. (1996). The intelligence advantage; Organizing for complexity. Boston: Butterworth-Heinemann.

McMaster, Tom, Vidgen, Richard, \& Wastell, David. (1998). Networks of association and due process in IS development. In Larsen, T., Levine, L., \& DeGross, J. (Eds.). (1999). Information systems: Current effects and future changes. Proceedings of the Joint IFIP WG 8.2 \& WG 8.6 Working Conference on Information Systems (pp. 341-357). Helsinki, Finland: IFIP, Laxenberg, Austria.

Mitchell, Alex \& Graham, Scott. (1995). Concurrency control in CSCW systems. January 16, 1995. Unpublished paper. See http://www.dgp.toronto.edu/people/alex/unpublished/concurrency.html

Nonaka \& Takeuchi (1995). The knowledge-creating company. New York, NY: Oxford University Press.

Redmiles, D. (1993). Reducing the variability of programmers' performance through explained examples. Human Factors in Computing Systems, INTERCHI '93 Conference Proceedings, ACM, April 1993, pp. 67-73.

Schein, Edgar H. (1999). The corporate culture survival guide: Sense and nonsense about culture change. San Francisco: CA, Jossey-Bass Publishers.

Schein, E. (1996). Three cultures of management: The key to organizational learning, Sloan Management Review, Fall, 9-20.

Schein, E. (1997). Organizational learning: What is new? Working paper, MIT Sloan School of Management. On the WWW at http://learning.mit.edu/res/wp/index.html

Sibbet, David, Drexler, Allan, \& The Grove Consultants International. (1994). Graphic guide to team performance. San Francisco, CA. The Grove Consultants International.

Somers, Pat, Rudman, Carrie \& Stevens, Clarke. (1997) Designing Web Interfaces for Realtime Collaboration - US WEST Advance Technologies. Presentation at the Third Conference on the Human Factors \& The Web, June 12, 1997, Denver, Colorado. http://www.uswest.com/web-conference/proceedings/somers.html

Stebbins, R. A. (1993). Social world, life-style, and serious leisure: Toward a mesostructural analysis. World Leisure and Recreation 35(1), Spring 1993, 23-26.

Wellins, Richard, Schaaf, Dick \& Harper Shomo, Kathy. (1994). Succeeding with teams: 101 tips that really work. Minneapolis, MN: Lakewood Books. 
Wheatley, Margaret J. (1999). Leadership and the new science revised: Discovering order in a chaotic world. Berrett-Koehler Publishers. 